\title{
Conocimiento sobre dengue en una región endémica de Perú. Estudio de base poblacional
}

\section{Knowledge about dengue in an endemic Peruvian region. A population- based study}

\author{
César Gutiérrez¹, Juan José Montenegro-Idrogo 2,3 \\ 1 Facultad de Medicina, Universidad de Piura. Lima, Perú. \\ 2 Facultad de Medicina, Universidad Nacional Mayor de San Marcos. Lima, Perú. \\ 3 Servicio de Medicina de Enfermedades Infecciosas y Tropicales, Hospital Nacional Dos de Mayo. Lima, Perú.
}

Correspondencia

Juan José Montenegro Idrogo

montenegroidrogo@gmail.com

\section{Recibido: 02/11/2017}

Arbitrado por pares

Aprobado: 06/12/2017

\section{Citar como: Gutiérrez C,}

Montenegro Idrogo JJ.

Conocimiento sobre dengue en una región endémica de Perú. Estudio de base poblacional. Acta Med Peru. 2017;34(4):283-8

\section{RESUMEN}

Objetivo: Describir los conocimientos sobre transmisión, sintomatología, acciones de prevención y control frente a dengue en la región Piura, Perú. Material y métodos: Análisis secundario de la sección 700 (salud) de la Encuesta Nacional de Programas Estratégicos 2014, realizada por el Instituto Nacional de Estadística e Informática a 113073 habitantes de $\geq 14$ años a nivel nacional (5 131 en Piura). Se analizaron las preguntas 701 al 704 sobre conocimientos de dengue. Las respuestas fueron analizadas según características demográficas y provincia de residencia. Además, se comparó los resultados de toda la región frente al promedio nacional. Resultados: En Piura, el $78,4 \%$ refirió que la transmisión de dengue es por la picadura de un mosquito, (solo 54,5\% a nivel nacional). Hubo diferencias entre zonas urbana (84\%) y rural $(58,2 \%)$, y entre provincias. Los síntomas más recordados fueron fiebre $(79,7 \%)$, cefalea $(56,4 \%)$, dolor de huesos/articulaciones $(30,3 \%$ ) y escalofríos (28,7\%). 96,9\% acudiría a un establecimiento de salud si presentara síntomas ( $97,8 \%$ a nivel nacional). Conocimiento sobre control de mosquito fue menor del $50 \%$ de medidas adecuadas. Conclusiones: El conocimiento sobre algunos aspectos del dengue es aún limitado en la región Piura, siendo ésta la más endémica a nivel nacional. Se debe enfatizar en educación sanitaria a nivel poblacional para frenar el avance alarmante de este problema.

Palabras clave: Dengue; Aedes aegypti; Enfermedades transmisibles emergentes; Conocimientos, actitudes y práctica en salud (fuente: DeCS BIREME).

\section{ABSTRACT}

Objective: To describe knowledge about dengue in an endemic Peruvian region (Piura). Materials and methods: Secondary Data analysis of the National Survey of Strategic Programs 2014 -section 700 (health) (Statistics and Informatics National Institute). 113073 national people surveyed at $\geq 14$ years old (5 131 surveyed in Piura). We to analyze questions 701 to 704 , about dengue knowledge. Answers to describe by demographics behavioral, residence zone and to compare with national results. Results: At Piura, $78.4 \%$ refer dengue to transmit by mosquito bite, but national results were $54.5 \%$. There are differences between urban zone (84\%) and rural zone $(58.2 \%)$; to there are differences between provinces. Symptoms frequently remembered: fever $(79.7 \%)$, headache (56.4\%), osteo-articular pain (30.3\%) and shivers (28.7\%). If symptoms present in people, $96.9 \%$ going to establishment health ( $97.8 \%$ nationally). Knowledge about actions for mosquito control was $<50 \%$. Conclusion: Knowledge about some dengue aspects yet is poor at Piura region- endemic dengue region nationally. Sanitary education should be empathizing in people, and back down the development of the disease.

Keywords: Dengue; Aedes aegypti; Communicable diseases, emerging; Health knowledge, attitudes, practice (source: MeSH NLM). 


\section{INTRODUCCIÓN}

El dengue es una enfermedad vírica transmitida por el mosquito de la especie Aedes spp ${ }^{[1]}$, que a su vez es vector de otras arbovirosis ${ }^{[2]}$ como chikungunya, fiebre amarilla, y actualmente zika ${ }^{[3]}$, todas ellas de relevancia en salud global debido a la mortalidad y/o complicaciones asociadas a estas enfermedades ${ }^{[1,2]}$.

Las actividades y programas de prevención junto con el control de vectores en zonas endémicas juegan un rol importante para evitar la dispersión de dengue y demás arbovirosis; no obstante, estas acciones suelen ser insuficientes ${ }^{[1,4-6]}$. El conocimiento del mecanismo de transmisión de las arbovirosis y del riesgo de la proliferación del vector podría permitir en la población la identificación temprana de focos de diseminación y evitar epidemias $^{[6,7]}$. A pesar de ello, los conocimientos en poblaciones de zonas de riesgo no suelen ser homogéneos ${ }^{[6-10]}$; reportándose cifras dispares en diferentes regiones a nivel nacional ${ }^{[8]} \mathrm{e}$ internacional. ${ }^{(9-13)}$ Este escenario debilita la identificación y acción oportuna ante estas enfermedades por parte de la población.

En Perú la enfermedad por virus del dengue es endémica en zonas de la costa norte y Amazonía peruana ${ }^{[14]}$. Piura, región ubicada en la costa norte del Perú y cercana al Ecuador, es un área endémica a nivel nacional, y expuesta a los efectos de las corrientes tropicales, que exacerban las condiciones de diseminación del vector en la región ${ }^{[15]}$. Llama la atención que a pesar de las medidas de prevención y control instauradas ${ }^{[17]}$, se evidencie un alarmante creciente número de casos notificados en la región. Así, en el año 2015 se notificaron 20561 casos de dengue que representó una incidencia anual de 11,15 por cada mil habitantes, siendo la región con mayor número de casos de dengue a nivel nacional en los últimos tres años y una de las regiones con mayor mortalidad por dengue (Figura 1$)^{[14]}$. Estas cifras motivaron que en mayo de 2017 sea considerada como una región con brote epidémico y de elevada mortalidad ${ }^{[18]}$. Este hecho genera una alerta, debido a la posibilidad de transmisión del resto de arbovirus trasmitidos por el mismo vector.

Por lo expuesto, el objetivo del estudio fue describir los conocimientos relacionados con la transmisión, sintomatología, acciones de prevención y control frente al dengue a partir de una encuesta poblacional en pobladores de la región Piura.

\section{MATERIALES Y MÉTODOS}

\section{Diseño}

Estudio descriptivo de análisis secundario de la sección 700 (salud) del apartado sobre dengue de la Encuesta Nacional de Programas Estratégicos 2014 (ENAPRES), realizada por el Instituto Nacional de Estadística e Informática (INEI) ${ }^{[16]}$.

Lugar y escenario epidemiológico actual de la región estudiada

Piura es una de las regiones localizada en la costa norte con variabilidad climática por la ocurrencia periódica del fenómeno de El Niño, que origina precipitaciones extraordinarias.
Tabla 1. Características generales de los encuestados en la región Piura, ENAPRES 2014.

\begin{tabular}{|c|c|c|c|}
\hline \multicolumn{2}{|c|}{ Características } & $\begin{array}{l}\text { Frecuencia } \\
\text { expandida }\end{array}$ & $\begin{array}{c}\% \\
\text { ponderado }\end{array}$ \\
\hline \multirow[t]{2}{*}{ Sexo } & Femenino & 710993 & 51,04 \\
\hline & Masculino & 681923 & 48,96 \\
\hline \multirow[t]{4}{*}{ Etario } & 14 a 24 & 223665 & 26,4 \\
\hline & 25 a 39 & 104515 & 24,2 \\
\hline & 40 a 59 & 123088 & 33,1 \\
\hline & 60 y más & 56344 & 19,3 \\
\hline \multirow[t]{8}{*}{ Provincia } & Piura & 547415 & 39,3 \\
\hline & Sullana & 236706 & 16,99 \\
\hline & Morropón & 161063 & 11,56 \\
\hline & Sechura & 102018 & 7,32 \\
\hline & Ayabaca & 95561 & 6,86 \\
\hline & Huancabamba & 95173 & 6,83 \\
\hline & Talara & 83093 & 5,97 \\
\hline & Paita & 71886 & 5,16 \\
\hline \multirow[t]{2}{*}{ Región natural } & Costa & 1266829 & 90,95 \\
\hline & Sierra & 126088 & 9,05 \\
\hline \multirow[t]{2}{*}{ Área } & Urbana & 1093547 & 78,51 \\
\hline & Rural & 299369 & 21,49 \\
\hline Total & & 1392916 & 100,0 \\
\hline
\end{tabular}

El clima semitropical de la costa norte es cálido, húmedo y con lluvias de verano, que se ve influenciado por la corriente del Niño; el subtropical-árido de la parte sur, bajo la influencia de la corriente peruana, presenta una temperatura ligeramente cálida, muy húmeda y sin lluvias. En la zona andina (sierra) de Piura el clima es templado-cálido en los pisos bajos andinos; como el de las provincias de Ayabaca y Huancabamba; y en los pisos medios (entre 2500 y los 3500 msnm.) es templado con lluvias regulares durante los meses de verano. El índice aédico reportado por DIGESA/DIRESA Piura en 2017 varía entre 3-30\% en los distritos de mayor presencia del vector, y las provincias con mayor incidencia de casos son Piura, Sullana, Morropón y Talara ${ }^{[14]}$.

\section{Población y muestra}

La encuesta de ámbito nacional (incluyó todas las regiones del país) entre enero y diciembre del año 2014; se entrevistaron 113073 habitantes de 14 años a más a nivel nacional (5 131 participantes en Piura/Población estimada INEI 2014: 1829496 habitantes). En la encuesta participaron todos los integrantes residentes 


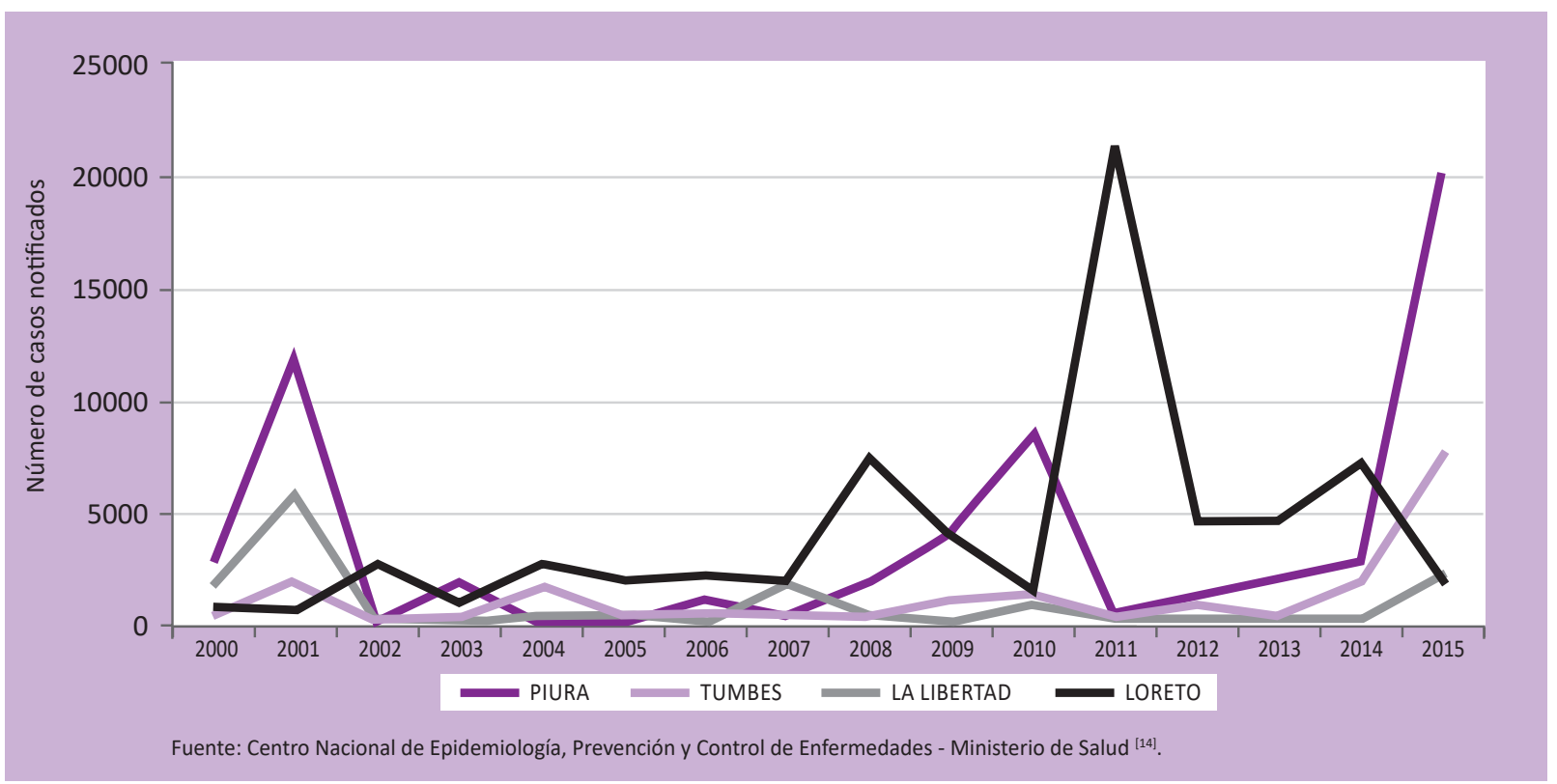

Figura 1. Evolución anual de los casos notificados de dengue en regiones con mayor prevalencia, Perú 2000-2015.

de la unidad familiar encuestada según las especificaciones metodológicas de la ENAPRES 2014. Se consideró el marco muestral básico de la información estadística y cartográfica del Censo 2007 actualizado al 2010 y 2011 del directorio de viviendas por conglomerados. La ENAPRES 2014 utilizó un muestreo probabilístico, estratificado, bietápico independiente por región y proporcional al tamaño poblacional ${ }^{[16]}$.

\section{Procedimiento y plan de análisis}

Se analizaron las preguntas 701 (¿cómo se transmite el dengue?), 702 (¿cuáles son los síntomas del dengue?), 703 (¿qué haría Ud. si presenta los síntomas del dengue) y 704 (¿qué haría Ud. para prevenir el dengue?). Las respuestas fueron analizadas según sexo de los encuestados, región natural (costa o sierra) y área de residencia (urbana o rural). Además, se comparó los resultados de toda la región frente al promedio nacional.

Para motivos del análisis se realizó el cálculo aplicando el factor de expansión de la muestra para la región Piura, que según el diseño de la ENAPRES toma en cuenta dos etapas, la unidad primaria de medida (conglomerado) y la unidad secundaria de medida (vivienda) ${ }^{[16]}$.

Se hizo uso del paquete estadístico IBM SPSS Statistics for Windows, version 21 (IBM Corp., Armonk, N.Y., USA) para todos los análisis.

\section{Aspectos éticos}

Las bases de datos de la Encuesta Nacional de Programas Estratégicos 2014 se encuentran disponibles de manera libre para su análisis y no contienen identificadores de las personas entrevistadas, garantizando la confidencialidad de la información. Es así que, en este tipo de análisis no es necesario la aprobación de un comité de ética para su ejecución.
Tabla 2. Conocimiento de síntomas atribuibles al dengue en la región Piura, ENAPRES 2014.

\begin{tabular}{lcc}
\multicolumn{1}{c}{ Síntomas de dengue } & $\begin{array}{c}\text { Frecuencia } \\
\text { expandida }\end{array}$ & $\begin{array}{c}\% \\
\text { ponderado }\end{array}$ \\
\hline Fiebre & 914571 & 79,7 \\
Dolor de cabeza & 646868 & 56,37 \\
Dolor de huesos y/o de & 347379 & 30,27 \\
articulaciones & 329059 & 28,68 \\
Escalofríos & 236026 & 20,57 \\
Náuseas y/o vómitos & 189910 & 16,55 \\
Sueño o decaimiento & 156622 & 13,65 \\
Diarrea & 115169 & 10,04 \\
Dolor muscular & 88875 & 7,75 \\
Dolor estomacal & 78624 & 6,85 \\
Sangrado & 41623 & 3,63 \\
Dolor de ojos & 35782 & 3,12 \\
Erupción cutánea & 63099 & 5,5 \\
Otro & 58618 & 5,11 \\
No sabe & & \\
\hline
\end{tabular}

\section{RESULTADOS}

\section{Características de la población}

El porcentaje de encuestados de sexo femenino (51\%) fue similar al de varones, las provincias con mayor número de encuestados fueron Piura, Sullana y Morropón, cabe mencionar que la encuesta se realizó en base al tamaño poblacional de cada región. La población predominante pertenece a la costa (91\%) y fue mayormente urbana (78,5\%) (Tabla 1$)$. 
Tabla 3. Conocimientos relacionados a dengue en la región Piura*, ENAPRES 2014.

\begin{tabular}{|c|c|c|c|c|c|c|c|c|}
\hline \multirow{2}{*}{ Conocimientos sobre dengue } & \multicolumn{2}{|c|}{ Sexo } & \multicolumn{2}{|c|}{ Región natural } & \multicolumn{2}{|c|}{ Área } & \multirow{2}{*}{$\begin{array}{l}\text { Región } \\
\text { Piura }\end{array}$} & \multirow{2}{*}{$\begin{array}{c}\text { Nivel } \\
\text { nacional }\end{array}$} \\
\hline & Masculino & emenino & Costa & Sierra & Urbana & Rural & & \\
\hline \multicolumn{9}{|l|}{ En su opinión, cómo se transmite (contagia) el dengue } \\
\hline Picadura de zancudo/mosquito & 77,19 & 79,58 & 81,9 & 42,12 & 84,04 & 58,18 & 78,42 & 54,5 \\
\hline Beber agua con larvas/huevos de zancudos & 11,78 & 13,63 & 13,58 & 3,89 & 14,06 & 7,92 & 12,73 & 17,3 \\
\hline Contacto persona a persona & 1,57 & 1,76 & 1,68 & 1,56 & 1,61 & 1,87 & 1,67 & 1,4 \\
\hline Otro & 1,63 & 1,85 & 1,65 & 2,73 & 1,5 & 2,61 & 1,74 & 2,6 \\
\hline No sabe & 15,9 & 13,23 & 10,65 & 54,94 & 8,42 & 36,54 & 14,53 & 28,5 \\
\hline \multicolumn{9}{|l|}{ Qué haría si presenta los síntomas del dengue } \\
\hline Acudir al establecimiento de salud & 96,18 & 97,56 & 97,02 & 94,58 & 96,84 & 97,33 & 96,92 & 97,8 \\
\hline Auto medicarse & 4,85 & 4,68 & 4,47 & 11,13 & 4,95 & 3,7 & 4,76 & 3,3 \\
\hline Tomar remedios caseros & 1,7 & 1,18 & 1,13 & 7,87 & 0,97 & 3,9 & 1,42 & 1,7 \\
\hline Tomar abundante líquido & 0,04 & 0,21 & 0,12 & 0,39 & 0,13 & 0,11 & 0,13 & 0,7 \\
\hline Otro & 0,13 & 0,05 & 0,09 & 0,0 & 0,03 & 0,44 & 0,09 & 0,5 \\
\hline No sabe & 0,37 & 0,48 & 0,41 & 0,78 & 0,46 & 0,22 & 0,43 & 0,3 \\
\hline \multicolumn{9}{|l|}{ Acciones que realizaría para prevenir el dengue } \\
\hline Tapar bien los recipientes donde almacena el agua & 46,65 & 57,88 & 53,76 & 27,05 & 55,04 & 39,43 & 52,53 & 45,4 \\
\hline $\begin{array}{l}\text { Lavar y escobillar los recipientes donde almacena } \\
\text { el agua }\end{array}$ & 23,45 & 32,26 & 28,61 & 16,65 & 29,33 & 21,46 & 28,06 & 30,8 \\
\hline $\begin{array}{l}\text { Desechar recipientes u objetos que puedan } \\
\text { contener (acumular) agua }\end{array}$ & 20,92 & 25,64 & 23,78 & 15,4 & 25,75 & 11,12 & 23,39 & 22,6 \\
\hline Utilizar mosquiteros para protegerse de las picaduras & 17,89 & 19,36 & 18,37 & 24,64 & 16,87 & 27,96 & 18,66 & 5,9 \\
\hline Fumigar la vivienda & 13,84 & 10,09 & 11,19 & 26,12 & 11,44 & 14,17 & 11,88 & 13,7 \\
\hline $\begin{array}{l}\text { Eliminar agua de lluvia acumulada en recipientes } \\
\text { y charcos }\end{array}$ & 9,26 & 8,37 & 8,59 & 12,99 & 9,29 & 6,22 & 8,79 & 16,0 \\
\hline $\begin{array}{l}\text { Utilizar la motita con larvicida entregada por el } \\
\text { personal de salud }\end{array}$ & 9,16 & 8,24 & 8,95 & 3,0 & 10,04 & 1,6 & 8,68 & 3,9 \\
\hline Otro & 23,16 & 28,73 & 25,86 & 30,57 & 27,1 & 20,7 & 26,07 & 21,7 \\
\hline No sabe & 9,0 & 4,56 & 6,26 & 15,43 & 5,54 & 12,61 & 6,68 & 4,4 \\
\hline
\end{tabular}

* Resultados expresados en porcentajes ponderados.

\section{Conocimientos sobre dengue}

En relación a los síntomas más frecuentemente respondidos que consideraron atribuibles al dengue fueron: fiebre $(80 \%)$, cefalea $(56,45 \%)$ seguidos de dolor de huesos y/o articulaciones, y escalofríos. En relación al sangrado solo fue reportado en $7 \%$ del total (Tabla 2).

En la pregunta ¿Cómo se transmite (contagia) el dengue?, el 78,4\% de la población respondió por medio de la picadura de mosquito/ zancudo. No obstante, cerca de $15 \%$ desconocía el mecanismo de transmisión, siendo más frecuente el desconocimiento en zona rural (36,5\%) y mucho más frecuente en las provincias de Ayabaca y Huancabamba con cifras cercanas al 50\%. En Sechura, Morropón y Paita el desconocimiento del mecanismo de transmisión del dengue fue más de $10 \%$ a diferencia de Talara, Piura y Sullana que reportaron menos porcentaje de desconocimiento del mecanismo de transmisión (Figura 2).

Cerca del $97 \%$ respondió que acudirían a un establecimiento de salud de presentar síntomas de dengue con frecuencias similares según región y zonas urbana y rural. Las acciones de prevención más frecuentes respondidas por la población fueron respectivamente: tapar recipientes donde se almacena el agua $(52,5 \%)$, lavar y escobillar recipientes donde se almacena el agua (28\%); y desechar recipientes que puedan contener (acumular) agua $(23,4 \%)$, además de otras respuestas en menor frecuencia. (Tabla 3). 


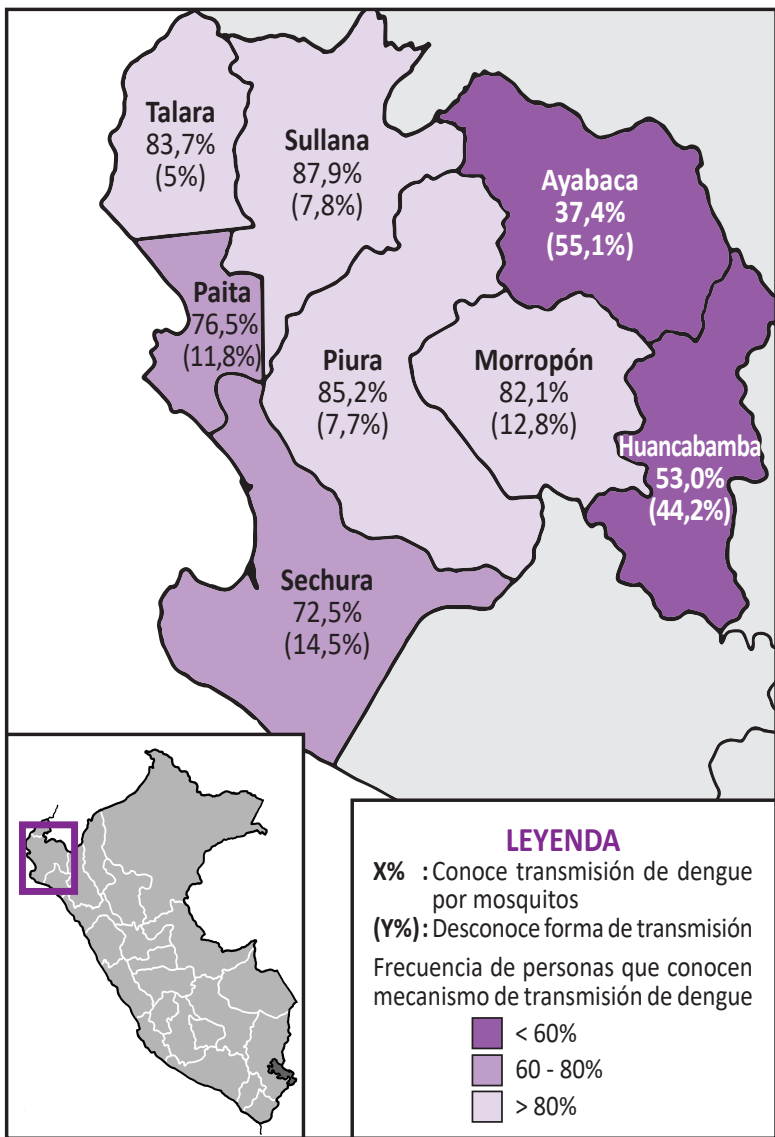

Figura 2. Conocimiento sobre la forma de transmisión del dengue según provincias de la región Piura, ENAPRES 2014.

\section{DISCUSIÓN}

El conocimiento sobre la forma de transmisión del dengue en la población de Piura (cerca de 80\%) no está alejado de lo reportado en otras áreas endémicas a nivel nacional como lquitos donde se evidenció que $85,6 \%$ de la población estudiada conocía el mecanismo de transmisión del dengue ${ }^{[8]}$. No obstante, llama la atención el porcentaje de desconocimiento sobre el mecanismo de transmisión que aumenta en determinadas zonas de la región hasta cerca del $50 \%$. Se menciona una problemática frente a las estrategias de prevención de dengue en zonas de la costa norte, donde hubo renuencia a los mecanismos de control vectorial, debido a que eran consideradas medidas inefectivas ${ }^{[17]}$. En Colombia, se ha reportado un bajo nivel de conocimiento del mecanismo de transmisión del dengue en la región de Bucaramanga, con cerca del $40 \%{ }^{\left[{ }^{[9]}\right.}$. En Brasil el conocimiento sobre el mecanismo de transmisión del dengue en un estudio reporta cifras mayores del $90 \%$ en la población ${ }^{[10]}$ con cifras similares en estudios en poblaciones de Australia ${ }^{[19]}$ y Costa Rica ${ }^{[11]}$. En países asiáticos se ha descrito un nivel de conocimiento entre $72 \%$ (India) y $88 \%$ (Malasia) ${ }^{[20,21]}$. Como se observa, los niveles de conocimientos son diversos y por ello es importante actualizar investigaciones al respecto y así tener elementos que permitan plantear estrategias de intervenciones de educación sanitaria comunitaria.
Al analizar el conocimiento por área de residencia, el desconocimiento de la forma de transmisión fue más frecuente en zona rural y en provincias de la sierra de la región Piura, observación consistente con otras investigaciones internacionales ${ }^{[17,20,21]}$, con excepción de la región Bucaramanga en Colombia ${ }^{[9]}$.

La mayoría de la población en el estudio refiere que acudiría a un establecimiento de salud ante si presenta síntomas de dengue; a diferencia de la población de Bucaramanga donde el $83 \%$ acudiría donde haya un profesional médico y $15 \%$ se automedicaría sin acudir a un centro de salud ${ }^{[9]}$. La frecuencia de síntomas más frecuentes que relacionan con dengue fueron fiebre, cefalea y otros síntomas inespecíficos similar a lo encontrado en Iquitos ${ }^{[8]}$ y otros estudios ${ }^{[10-13,19-22]}$. La presencia frecuente de síntomas aumentaría la sospecha de dengue en la población, y el acudir al establecimiento ante esta sospecha influiría en captación temprana de casos probables. No obstante, no se especifica el tiempo en el que acude al establecimiento.

Las acciones para prevenir el dengue que frecuentemente fueron contestadas guardaban relación con los cuidados de los recipientes de agua y en menor frecuencia el uso de insecticidas y mosquiteros. Estos resultados guardan relación con los problemas encontrados por Palma-Pinedo y col, donde las medidas de control vectorial no eran aceptadas en pobladores de la costa norte, debido a que las consideraban poco efectivas e incomodas ${ }^{[17]}$. Paz-Soldán y col en un estudio poblacional con 1333 pobladores de Iquitos describió cifras similares sobre medidas de prevención y control vectorial, donde el uso de mosquiteros es de $53 \%$ y medidas como fumigación o uso de productos químicos es menor al $20 \%$; más alarmante aún las medidas relacionadas con recipientes de agua son menores del $40 \%$. Además, menos de $20 \%$ refirieron conocer el comportamiento diurno del mosquito (picaba en horas del día) ${ }^{[8]}$ cifras similares que Tailandia ${ }^{[13]}$, pero diferentes de las poblaciones de Colombia ${ }^{[0,10]}$ y Brasil ${ }^{[10]}$ donde es frecuente ( $81 \%$ y $96 \%$ respectivamente) el control y lavado de recipientes de agua.

El presente estudio al utilizar bases de datos secundarias procedentes de estudios poblacionales con factores de ponderación, se limita a la descripción valorada según estos parámetros, que si bien procuran ser representativos de la región no están diseñados inicialmente para el tema en estudio.

Piura, a pesar de ser una región endémica de dengue y Aedes con cifras alarmantes los últimos años de casos notificados de dengue, los conocimientos sobre transmisión y control de dengue en su población no son homogéneos en toda la región, además en relación a control del vector el conocimiento es bajo (20$50 \%)$, es necesaria la intervención y educación en prevención y control de dengue y arbovirus asociados al Aedes en la población de las localidades más vulnerables de la región, donde estos conocimientos no son adecuados. 
Fuente de financiamiento: El autor declara no haber recibido ninguna financiación para la realización de este trabajo.

Declaración de conflicto de intereses: Los autores declaran no tener conflicto de intereses con la publicación de este artículo.

\section{REFERENCIAS BIBLIOGRÁFICAS}

1. Guzman MG, Harris E. Dengue. Lancet. 2015;385(9966):453-65.

2. Rey JR, Philip Lounibos P. Ecología de Aedes aegypti y Aedes albopictus en América y transmisión enfermedades. Biomédica. 2015;35:177-85.

3. Bouyer J, Chandre F, Gilles J, Baldet T. Alternative vector control methods to manage the Zika virus outbreak: more haste, less speed. Lancet Glob Health. 2016;4(6):e364

4. Hermann LL, Gupta SB, Manoff SB, Kalayanarooj S, Gibbons RV, Coller BA. Advances in the understanding, management, and prevention of dengue. J Clin Virol. 2015;64:153-9.

5. Bhatt S, Gething PW, Brady OJ, Messina JP, Farlow AW, Moyes $\mathrm{CL}$, et al. The global distribution and burden of dengue. Nature. 2013;496:504-507.

6. Quintero J, Brochero $\mathrm{H}, \mathrm{Manrique-Saide} \mathrm{P}, \mathrm{Barrera}-$ Pérez $\mathrm{M}$, Basso C, Romero S, Petzold M, et al. Ecological, biological and social dimensions of dengue vector breeding in five urban settings of Latin America: a multi-country study. BMC Infect Dis. 2014;21:14:38.

7. Kroeger A, Lenhart A, Ochoa M, Villegas E, Levy M, Alexander N, et al. Effective control of dengue vectors with curtains and water container covers treated with insecticide in Mexico and Venezuela: cluster randomised trials. BMJ. 2006;332:1247-1252.

8. Paz-Soldán VA, Morrison AC, Cordova Lopez JJ, Lenhart A, Scott TW, Elder JP, et al. Dengue Knowledge and Preventive Practices in Iquitos, Peru. Am J Trop Med Hyg. 2015;93(6):1330-7.

9. Cáceres-Manrique FM, Vesga-Gómez C, Perea-Florez X, Ruitort M, Talbot Y. Conocimientos, Actitudes y Prácticas sobre Dengue en Dos Barrios de Bucaramanga, Colombia. Rev. salud pública. 2009;11(1):27-38

10. Santos SL, Parra-Henao G, Silva MB, Augusto LG. Dengue in Brazil and Colombia: a study of knowledge, attitudes, and practices. Rev Soc Bras Med Trop. 2014;47(6):783-7.

11. Egedus VL, Ortega JM, Obando AA. Knowledge, perceptions, and practices with respect to the prevention of dengue in a mid-Pacific coastal village of Costa Rica. Rev Biol Trop. 2014;62(3):859-67.
12. Wong LP, AbuBakar $S$. Health beliefs and practices related to dengue fever: a focus group study. PLoS Negl Trop Dis. 2013;7(7):e2310.

13. Van Benthem BH, Khantikul N, Panart K, Kessels PJ, Somboon P, Oskam L. Knowledge and use of prevention measures related to dengue in northern Thailand. Trop Med Int Health. 2002;7(11):9931000

14. Sala de Situación de Salud - Semana Epidemiológica N 112017 [Internet]. Lima: Centro Nacional de Epidemiología, Prevención y Control de Enfermedades - Ministerio de Salud; 2017 [citado el 10 de octubre de 2017]. Disponible en: http://www.dge.gob.pe/ portal/docs/vigilancia/sala/2017/salaSE11.pdf.

15. Ferreira MC. Geographical distribution of the association between El Niño South Oscillation and dengue fever in the Americas: a continental analysis using geographical information system-based techniques. Geospat Health. 2014;9(1):141-51.

16. Encuesta Nacional de Programas Estratégicos 2011-2014 [Internet]. Lima: Instituto Nacional de estadística e Informática; 2015 [citado el 10 de octubre de 2016]. Disponible en: https://www.inei.gob. pe/media/MenuRecursivo/publicaciones_digitales/Est/Lib1291/ libro.pdf

17. Palma-Pinedo $H$, Cabrera R, Yagui-Moscoso $M$. Factors behind people's reluctance towards dengue vector control actions in three districts in northern Peru. Rev Peru Med Exp Salud Publica. 2016;33(1):13-20.

18. OMS habla de una epidemia por dengue en región Piura [Internet]. Lima: CMP noticias; 2016 [citado el 10 de octubre de 2016]. Disponible en: https://cmp.org.pe/oms-habla-de-una-epidemiapor-dengue-en-la-region-piura/

19. Gyawali N, Bradbury RS, Taylor-Robinson AW. Knowledge, attitude and recommendations for practice regarding dengue among the resident population of Queensland, Australia. Asian Pac J Trop Biomed. 2016;6(4):360-366.

20. Malhotra G, Yadav A, Dudeja P. Knowledge, awareness and practices regarding dengue among rural and slum communities in North Indian city, India. Int J Med Science and Public Health. 2014;3(3):295-299.

21. Hairi F, Ong CH, Suhaimi A, Tsung TW, Sundaraj C, Soe MM, et al. A knowledge, attitude and practices (KAP) study on dengue among selected rural communities in the Kuala Kangsar district. Asia Pac J Public Health. 2003;15(1):37-43.

22. Dhimal M, Aryal KK, Dhimal ML, Gautam I, Singh SP, Bhusal CL, et al. Knowledge, attitude and practice regarding dengue fever among the healthy population of highland and lowland communities in central Nepal. PLoS One. 2014;9(7):e102028. 